\title{
Water drop movement on woven fiber mat surfaces due to flow of diesel fuel
}

\author{
Jia Fang ${ }^{1}$, Abdulwahab. Aljuhani ${ }^{1,2}$, GeorgeG.Chase ${ }^{1}$ \\ 1. Department of Chemical and Biomolecular Engineering \\ ${ }^{1}$ The University of Akron, Akron, OH, 44325 \\ ${ }^{2}$ Yanbu Industrial College, Yanbu, Saudi Arabia
}

\begin{abstract}
Fuel contamination by water is one of the major factors of engine failures. Separation of water from diesel fuel is often achieved by coalescing or depth filter media. The separation performance is strongly related to the motion of drops on the surface and in the depth of a filter medium. The dynamics of the motions of drops on a filter surface are influenced by many factors such as the wetting properties of the media. Direct observations of drop movements on fiber surfaces are not well documented. Of particular interest in this work are the dynamics of drops on surfaces of hydrophobic mats that resist drop movement into the interior of the mat, forcing the drops to move on the surface of the mat. Such materials function as barriers to the dispersed phase and allow the continuous phase to move through the mat.
\end{abstract}

In this paper, the motions of water drops were studied on surfaces of mats woven of polypropylene, nylon and Teflon ${ }^{\mathrm{TM}}$ (ie, polytetrafluoroethylene) fibers. Pore sizes of the mats ranged from 100 to 1000 microns and drop sizes ranged from 200 to 6000 microns. The flow of ultra low sulfur diesel fuel parallel to the mat surface provided a drag force that induced the movement of the drops. The effects of surface wettability, flow velocity, drop size, fiber size, fiber mat pore size, fiber mat orientation were considered. A correlation for a drag coefficient to estimate the average velocity of drops moving on the woven mats surfaces was derived from the flow and the material's characteristics. In addition, another correlation was obtained for estimating the minimum velocity, or minimum Reynolds number, required to initiate the drop motion on the woven mat surfaces.

Key word: woven fiber mat, drag force, drag coefficient, drop motion

Corresponding Author: gchase@uakron.edu, 330.972.7943 


\section{Introduction}

The interactions between water drops and hydrophobic woven fiber mats are of interest in many applications such as separation of water drops from oil, dehumidification, and rain coats [1]. Depending on the applications, the materials, the mat structures, and the fluid flow directions, the drops may pass through a mat or move across the surface of a mat. In this work the movements across hydrophobic mats by water drops submerged in diesel fuel are investigated. The motivation of this study is to create better understanding of hydrophobic membranes to separation of water drops from oil.

The presence of water in diesel fuels in the form of droplet dispersions can cause many problems such as plugging of fuel injectors, reduction of fuel flow rate, reduction of fuel lubricity and corrosion of engine parts [2]. Hence, removing water contamination from diesel fuel is important to the maintenance and life of diesel engines. When a water drop size is larger than 100 microns, the water drops can be effectively removed by gravity settling [3]. However, dispersions of drops smaller than 100 microns are more difficult to separate and often require the use of coalescing depth filters [4] or a membrane technology [5].

Madani et al. [6] observed that the motion of a drop on flat surface can be characterized as different behavioral regimes a) sliding, b) deforming, and c) detachment, depending on the drop size, the drop fluid properties, the continuous fluid phase properties, and the flow conditions. Under appropriate conditions, water drops easily roll or slide across the hydrophobic surfaces and leave behind little to no residue [7, 8]. Daniel and Chaudhury [9] found that drops on surfaces with spatially varying wettability tend to move toward the more wetting part of the surface with speeds of 1-2mm/s on a gradient surface. Subramanian et al. [10] developed estimates the speed of drops on surfaces with a wettability gradient. Gather et al. [11] observed water drops moving by gravity on inclined hydrophobic surfaces. Sheng and Zhang [12] investigated water drop motions on ratchet-like superhydrophobic surfaces. Bhushan and Jung [13] observed the static contact angles on the patterned silicon surfaces and measured adhesive forces and coefficient of friction with the help of atomic force microscope. However, to our knowledge, correlations for the motions of drops across woven mats due to the drag force by liquid flow have not been reported. 
The separation efficiency of a filter is strongly related to the dynamic motions of drops in a filter medium. Fundamental drop motions in a filter include movements of drops on single fibers, at junctions of two fibers, on mat surfaces, through pores of a thin mat, and with the depth of a thick fiber mat. The motion of drops on the surface of a filter is relevant to understanding the rate of liquid drainage from a filter. Drops draining from the filter are typically much larger than the emulsified drops, with sizes ranging from tenths to tens of millimeters in diameter.

In this work, the movements of water drops across surfaces of woven mats of polypropylene (PP), polyamide (nylon), and polytetrafluroethylene (PTFE) fibers were observed due to the drag flow of ultra low sulfur diesel (ULSD) fuel. The water droplets moved when the ULSD drag force exceeded the motion resistance forces that tended to hold the drops stationary. The ULSD flow rate was gradually increased until water drop movement was observed. The minimum ULSD velocity, or minimum Reynolds number, at which the drop began to move, was fitted to a correlation for predicting conditions to initiate drop movement. The initial drop motion depended on the surface properties of the fiber mats, such as wettability, surface roughness and heterogeneities [14]. Another correlation was fitted to relate the drag coefficient to the Reynolds and Capillary numbers of a drop moving on the mat surfaces. This work only considered clamshell shaped drops of sizes ranging from about 200 microns to 6000 microns that sat on top of the surface of the mats and did not penetrated into the fiber mats. The drops were two to six times larger than the mat pore openings.

\section{Description of experiments}

\subsection{Mat, liquid materials, equipment}

Woven PP mats had squared shaped pore opening with sides of length 105, 210, 500 and $1000 \mu \mathrm{m}$ (referred as samples PP105, PP210, PP500 and PP1000, respectively) were purchased from Spectrum Labs. The nylon and PTFE mats had parallelogram shaped openings. The diagonal lengths were $1080 \mu \mathrm{m}$ and $490 \mu \mathrm{m}$ for the nylon mats and $890 \mu \mathrm{m}$ and $520 \mu \mathrm{m}$ for the PTFE mat. The mats were cleaned prior to the experiments by sonication in distilled water, and dried in an oven to remove moisture. The thin glass fiber media (used as laminarization media) were supplied by Hollingsworth \& Vose Company. 
The liquids used in this study were ULSD (purchased locally) and deionized water (DI water). Some of the properties of these two liquids are summarized in Table 1. The equipment used in the experiments included a custom made Plexiglas holder, a flow meter (Cole Parmer, 03410ST), a pump (Fasco Industries, Inc), a CMOS high-speed camera (Mikrotron MC 1310), a drop shape analyzer with camera(DSA20E Krüss GmbH), microscope (OLYMPUS, SZ-PT), and spin coater (Laurell technologies corporation, WS-400B-6NPP/LITE/AS).

Table 1 . Physical properties of reference liquids at $20^{\circ} \mathrm{C}$.

\begin{tabular}{|l|c|c|c|c|}
\hline Liquid(Source) & $\begin{array}{l}\text { Density } \\
\left(\mathrm{Kg} / \mathrm{m}^{\wedge} 3\right)\end{array}$ & $\begin{array}{l}\text { Viscosity } \\
(\mathrm{cP})\end{array}$ & $\begin{array}{l}\text { Surface tension } \\
(\mathrm{mN} / \mathrm{m})\end{array}$ & $\begin{array}{l}\text { Boiling point } \\
\left({ }^{\circ} \mathrm{C}\right)\end{array}$ \\
\hline ULSD (Purchased locally) & 870 & 1.4 & 29.83 & $180-360$ \\
\hline $\begin{array}{l}\text { DI water (Obtained by ion } \\
\text { exchange, 0.1 Siemens) }\end{array}$ & 1000 & 1.02 & 73.5 & 1000 \\
\hline
\end{tabular}

\subsection{Experiment setup}

Figure 1 shows a diagram of the pressure-driven flow device. ULSD was pumped through a flow meter and into a thin-slit channel holding the test mat and the water droplets. The body and cover of the holder were machined from Plexiglas. The flow channel was $4 \mathrm{~mm}$ deep, $3.5 \mathrm{~cm}$ wide, and $9 \mathrm{~cm}$ long. The test mats were positioned on the bottom of the channel and secured in place using an acrylic tape. To distribute the flow over the cross section of the channel, a glass fiber mat was positioned near the inlet of the channel as a flow-laminarization mesh. 


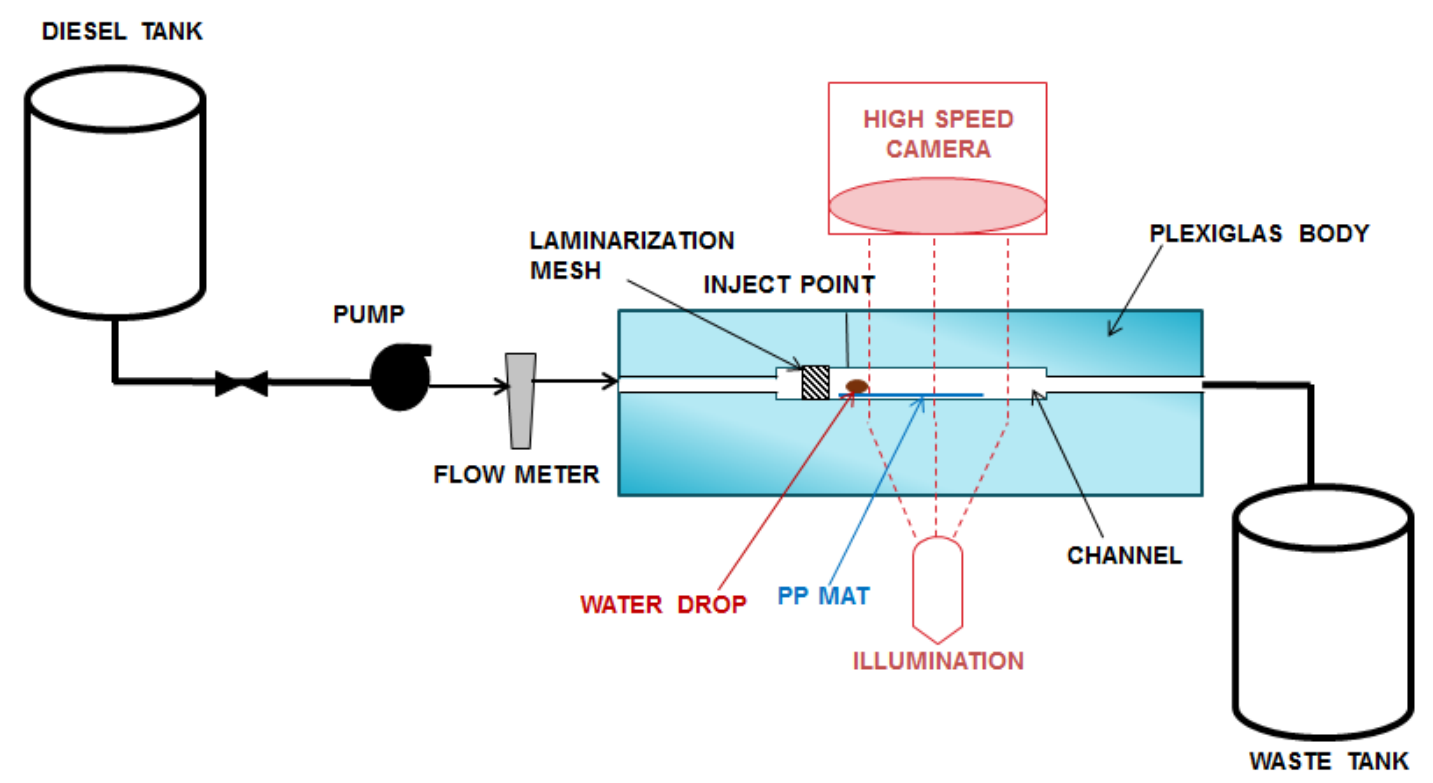

Figure 1 Flow diagram of the experiment

A water droplet was injected with a calibrated syringe and needle into the channel and onto the sample may by inserting the needle through a small port located on the top cover. With the help of the calibrated scale of the syringe, the drop volume and size was controlled. After injecting the water drop, the injection window was sealed by an acrylic tape. The channel was illuminated from below, and the movements of the drops on the woven mats were recorded by using the high-speed camera from above. Simultaneously, the movements of the drops also were recorded from a side view by the camera of the DSA20E.

\subsection{Experiment plan}

Due to the structure of the woven mats, the orientation of the weave relative to the flow direction affected the drop dynamic. The mat orientation was characterized by the acute angle formed between one of the fibers in the mat structure and the ULSD flow direction as the mat was rotated as a rigid body, preserving the structural angles between crossed fibers within the mats. Six different angles ( $\alpha=0,24,30,45,60$ and $66 \mathrm{deg})$ were evaluated. Figure 2 shows example images taken from the high speed camera positioned above the mats and water drops of the three different mat materials and the different angles. The ULSD flow direction was horizontal from left to the right for the images in Figure 2. 
The experimental observations showed that the velocities of drops whose contact areas with the mats were about the same size as the pore area (or drops with diameters $d_{A}$ about the same as the dimension of the pore opening) were affected by the mat fiber angle $\alpha$. Smaller drops tended to either follow the axis of a fiber or follow the channel between two fibers, until an irregularity in the mat structure caused the drop velocity direction to change. Drops greater than about 5 times the pore diameter tended to move across the mat in the flow direction independent of the mat orientation. The experiments in this work considered drop/pore diameter ratios from about 2 to 50. Pore diameters of the mats ranged from about 100 to 1000 microns and drop sizes ranged from about 200 to 6000 microns. The diameter ratio and the mat orientation angle $(\cos \alpha)$ were included in the data analysis. The correlations were fitted to the velocity components of the drops in the direction of the fluid flow. 

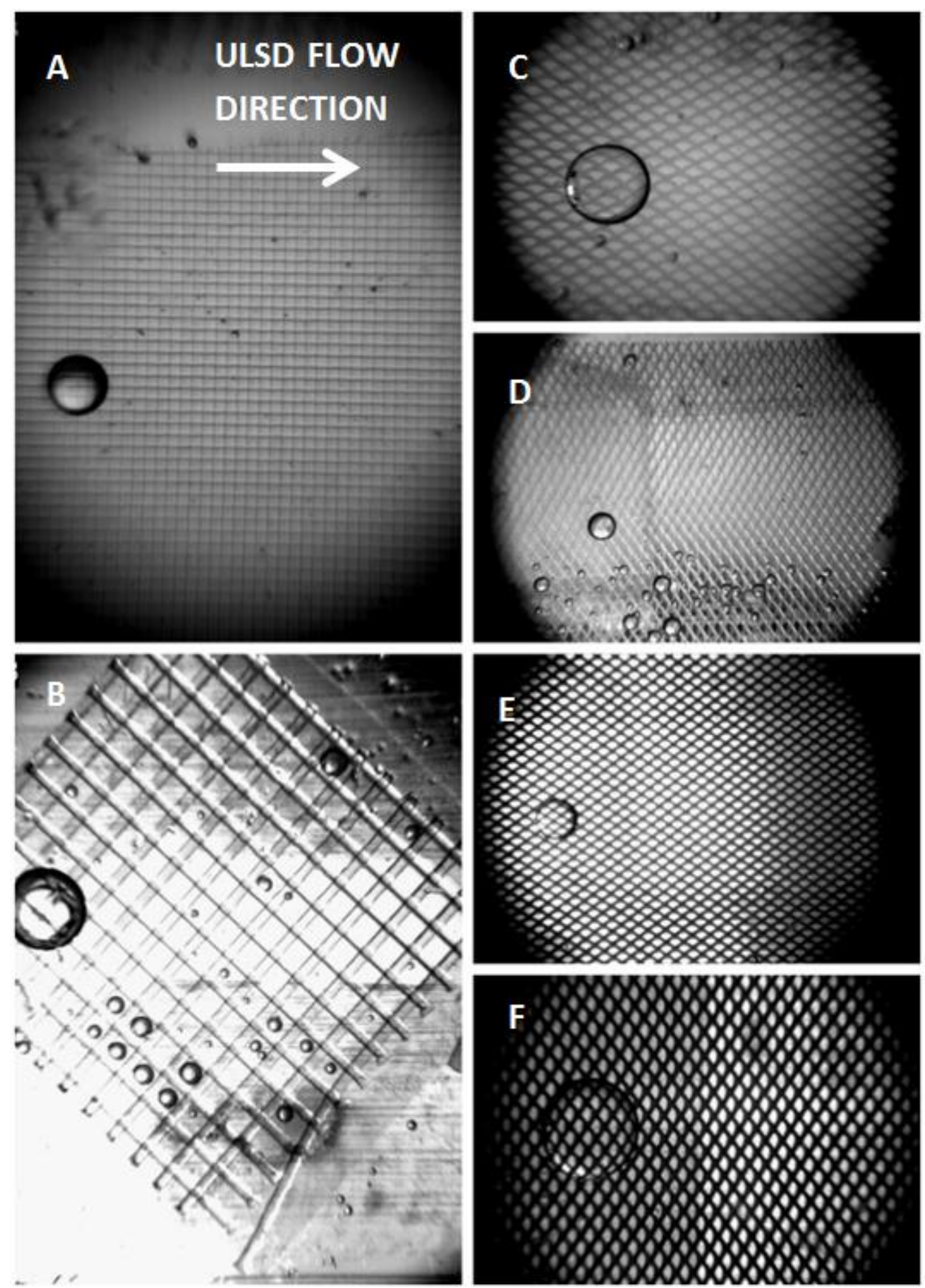

Figure 2. Example images of top views of water droplets on different mat surfaces with different angles relative to the flow direction: A) 0 degree PP mat, B) 45 degree PP mat, C) 24 degree nylon mat, D) 66 degree nylon mat, E) 30 degree PTFE mat F) 60 degree PTFE mat. 


\section{Data analysis}

\subsection{Correlation for drag coefficient of drop moving on fiber mat}

Figure 3 shows a sketch of a drop moving on a hypothetical woven mat at velocity $U_{\text {water }}$ due to the drag force of the ULSD flowing at velocity $V_{U L S D}$ parallel to the mat surface. The mat structure is represented by fibers oriented in the plane of the diagram and perpendicular to the plane, as expected of a square woven mat of fibers. The mat structure has peaks and valleys over which the drop moves. However, the model considers the mat surface to be flat and the effects of the peaks and valleys are accounted for by the values of the fitted parameters. The sketch shows the drop size moderately larger than the distance between the fibers, but in the experiments many of the drop sizes were much larger than the distance between the fibers and the effects of the peaks and valleys on the motions of the larger drops were less pronounced.

Figure 4 shows the horizontal force balance on a drop on a hypothetical flat mat surface as the ULSD flows left to right. The general force balance relates the acceleration of the drop $\frac{d M U}{d t}$ to the drag force between ULSD and the water drop $\left(F_{D}\right)$, the drag force between the water drop and mat $\left(F_{m a t}\right)$, and the contact line force $\left(F_{c l}\right)$.

$\frac{d M U}{d t}=F_{D}-F_{m a t}-F_{c l}$

The drop rapidly accelerates due to the ULSD flow, but because of the periodic structure of the fiber mat, the drop motion becomes oscillatory about a time averaged value. Since the interest is in the drop motions on a long time scale (relative to the time period of the oscillatory motion) and over long distances (relative to the pore size of the mat) the model is treated as a timeaverage pseudo steady state motion. Hence, Eq (1) reduces to

$$
F_{D}=F_{m a t}+F_{c l}
$$






Figure 3. Schematic of a water droplet moving on a woven PP mat at a velocity $U_{\text {water }}$ due to the drag of the ULSD flowing at velocity $V_{U L S D}$ parallel to the mat surface.



Figure 4. Diagram of the horizontal forces acting on a drop moving on a surface. $\theta \mathrm{r}$ and $\theta \mathrm{a}$ represent the advancing and receding contact angles, respectively. The arrows indicate the directions of the drag force of ULSD on the drop $\left(F_{D}\right)$, the drag force between the drop and the fiber mat (Fmat), and the contact line force due to the imbalance in the advancing and receding contact angles that resists the drop movement $(F c l)$. 
According to the definition of the drag force, the drag force acting on the drop due to the ULSD flow is equal to $[15,16]$,

$F_{D}=C_{D} A_{\text {drop }} \frac{1}{2} \rho_{\text {diesel }}(V-U)^{2}$

where $A_{d r o p}=\frac{\pi}{4} d_{L}^{2}$ is the projected area of the drop in the direction of the ULSD flow.

$C_{D}$ is the drag coefficient, and $U$ and $V$ are the drop and diesel fuel velocities, respectively. The drag force acting between the drop and mat is given as,

$F_{\text {mat }}=C_{\text {mat }} A \frac{1}{2} \rho_{\text {water }} U^{2}$

where $A$ is the contact area between the droplet and mat, and can be calculated by $A=\frac{1}{4} \pi d_{c}^{2}$ and $d_{c}$ is the diameter of the contact area between the drop and the mat. The contact line force is equal to $[17,18]$,

$F_{c l}=d_{c} \gamma_{w a t e r}\left(\cos \theta_{r}-\cos \theta_{a}\right)$

where $\gamma_{\text {water }}$ is the surface tension of the water, $\theta_{r}$ and $\theta_{a}$ are the receding and advancing angles on the two ends of the droplet.

Equations (2) - (5) are combined and rearranged to obtain and expression for the drag coefficient for the drag force between the drop and the mat, as

$C_{\text {mat }}=\frac{C_{D}\left(\frac{\pi}{4} d_{L}^{2}\right) \frac{1}{2} \rho_{\text {diesel }}(V-U)^{2}-d_{c} \gamma_{\text {water }}\left(\cos \theta_{r}-\cos \theta_{a}\right)}{\frac{1}{4} \pi d_{c}^{2} \frac{1}{2} \rho_{\text {water }} U^{2}}$

When the advancing and receding contact angles are approximately the same, as observed in our data described in the experimental section, the surface tension force term in the numerator of the right hand side term of Eq.(6) is neglected. Though this term is neglected, the static contact angle still contributes to the value of $C_{m a t}$ in the correlation. Also, the difference between advancing 
and receding contact angles are believed to contribute to the tendency of drops to not move until the gas flow rate exceeds a minimum magnitude. The drag coefficient for the ULSD flowing around the droplet was estimated from the correlation for fluid drag around a sphere [19],

$$
C_{D}=\frac{24}{\operatorname{Re}_{\text {diesel }}}\left(1+0.14 \operatorname{Re}_{\text {diesel }}^{0.7}\right) \quad\left(1 \leq \mathrm{Re}_{\text {diesel }} \leq 1000\right)
$$

Dimensional analysis [20,21] was applied to identify the independent dimensionless numbers on which the drag coefficient depends. Experimental data were fitted to determine the parameters in the correlation.

The drag force Fmat between a moving water drop and the fiber mat was related to the kinetic energy through Eq. (4) and the drag coefficient, $C_{m a t}$. Through experience, the drag force is expected to be a function of independent material, geometric, and process variables, namely,

$$
F_{\text {mat }}=F_{\text {mat }}\left(\rho_{\text {water }}, \mu_{\text {water }}, U, d_{A}, d_{f}, d_{\text {pore }}, \gamma_{\text {water }}, \cos \alpha\right)
$$

The drag coefficient, $C_{m a t}$, is assumed to be a power-law function of the above independent variables. The dimensionless Reynolds and Capillary numbers

$$
\begin{aligned}
& \operatorname{Re}_{\text {mat }}=\frac{\rho_{\text {water }} d_{\text {pore }} U}{\mu_{\text {water }}} \\
& C a=\frac{\mu_{\text {water }} U}{\gamma_{\text {water }}(1+\cos \theta)}
\end{aligned}
$$

are introduced into the power-law expression and dimensionless geometric ratios are defined. The dimensionless quantities are raised to an empirical parameter.

The drag coefficient $C_{m a t}$ has the assumed functional form:

$$
C_{\text {mat }}=A \operatorname{Re}_{\text {mat }}^{B} C a^{C}\left(\frac{d_{A}}{d_{\text {pore }}}\right)^{D}\left(\frac{d_{f}}{d_{\text {pore }}}\right)^{E}(\cos \alpha)^{F}
$$

where parameters $A, B, C, D, E, F$ must be determined from the experimental data. 


\subsection{Correlation for minimum Reynolds number for drop movement}

All of the drops moved on the mats when exposed to a high enough flow rate of ULSD. The resistance to the drop movement at lower flow rates was attributed to the surface roughness or irregularities in the surface properties. A correlation for predicting the lowest Reynolds number to initiate the drop motion can be useful in some applications.

The probability of a drop moving or not on a fiber mat was considered to be a function of the relevant variables,

Probability $=f\left(\rho_{\text {diesel }}, \mu_{\text {diesel }}, \sin \theta, d_{L}, d_{f}, d_{\text {pore }}, \gamma_{\text {water }}, V_{\text {diesel }}, \rho_{\text {water }}, \mu_{\text {water }}, \cos \alpha\right)$

In this application only data from drops that moved were considered and the corresponding diesel flow velocities when the drops first moved. The velocities were determined by gradually increasing the diesel flow rates. Hence the probability is unity (1). The dependencies of the variables were assumed to be a power law form. The probability function had the form

$1=k \rho_{\text {diesel }}^{a} \mu_{\text {diese }}^{b} \sin \theta^{c} d_{L}^{d} d_{f}^{e} d_{\text {pore }}^{f} \gamma_{\text {water }}^{g} V_{\text {diesel }}^{h} \rho_{\text {water }}^{i} \mu_{\text {water }}^{j} \cos \alpha^{l}$

where the coefficients $a, b, \ldots, l$ are constants. The Reynolds number of the ULSD around the drop, and Laplace numbers are defined by

$\operatorname{Re}_{U L S D \min }=\frac{\rho_{\text {diesel }} d_{L} V_{\text {diesel }}}{\mu_{\text {diesel }}}$

$L a=\frac{\rho_{\text {water }} d_{\text {pore }} \gamma_{\text {water }}}{\mu_{\text {water }}^{2}}$

where $R e_{U L S D \text { min }}$ is the ratio of the ULSD inertial forces to the viscous drag forces and $L a$ is the ratio of the interfacial forces to the viscous drag force between the drop and the mat.

Substituting $\operatorname{Re}_{U L S D \text { min }}$ and $L a$ into Eq.(13), rearrangement of the equation into dimensionless form, yields the working correlation of the form 
$\operatorname{Re}_{\text {ULSD min }}=H L a^{I}(\sin \theta)^{J}\left(\frac{d_{L}}{d_{\text {pore }}}\right)^{K}\left(\frac{d_{f}}{d_{\text {pore }}}\right)^{L}\left(\frac{\rho_{\text {diesel }}}{\rho_{\text {water }}}\right)^{M}\left(\frac{\mu_{\text {diesel }}}{\mu_{\text {water }}}\right)^{N}(\cos \alpha)^{O}$

where $H, I, \ldots O$ are constants to be determined from experimental data. $R e_{U L S D \min }$ is the minimum ULSD phase Reynolds number needed to initiate drop motion.

\section{Results and discussion}

\subsection{Wettability Results}

The contact angles of water drops on woven mat submerged in ULSD are summarized in Table 2. All drop volumes were $5 \mu \mathrm{L}$. Four example images of water drops on PP/nylon/PTFE mats are shown in Figure 5. All of the drops were clamshell shaped. The differences in the drop diameters were insignificant with drop diameter ratios in the range of $0.9<\frac{d_{L}}{d_{A}}<1.0$ due to the high contact angles with the surfaces and the small drop sizes. The contact angle were similar for the different mat materials because the fibers were all hydrophobic and the ULSD filled the pores. The edge lengths of the pores of the PP mats were verified and the fiber diameters were measured from optical microscope images, such as shown Figure 6. The equivalent pore diameters of the nylon and PTFE mats were calculated from the projected surface areas of the parallelogram shaped pores. 

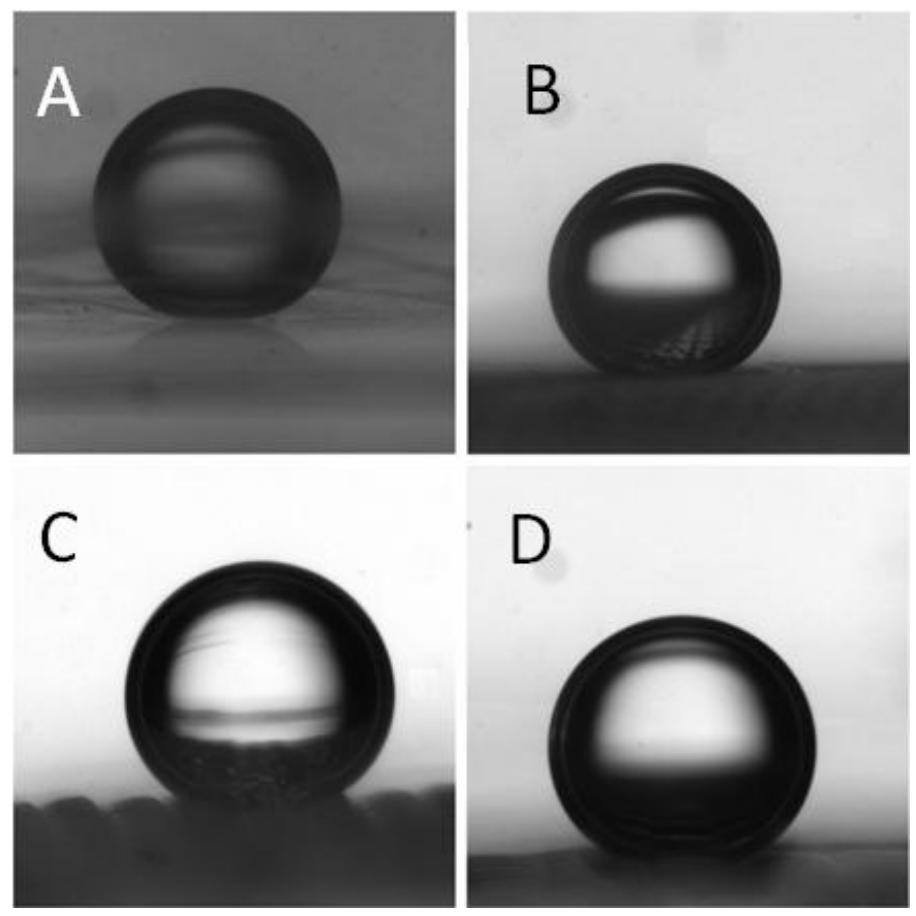

Figure 5 DSA20E photographs of clamshell shaped water drops with the same volume on different mat surfaces in diesel, (A) flat solid PP surface (B) PP210 (pore size $105 \mu \mathrm{m}$ ) (C) Nylon (pore size $785 \mu \mathrm{m}$ ) (D) PTFE (pore size $705 \mu \mathrm{m}$ ). The images are at different magnifications and hence are not comparable in scale.

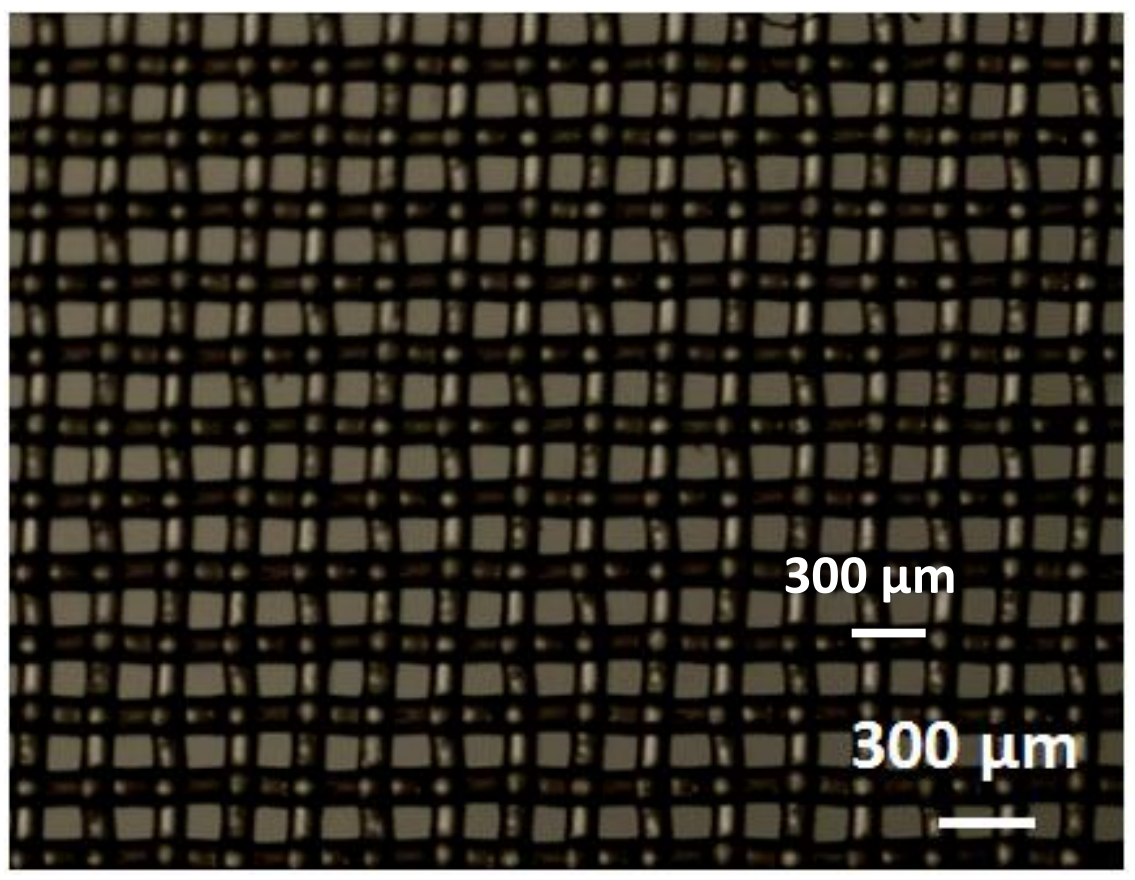

Figure 6. Example microscope image of mat PP105. 
Table 2. Summary of properties of the fiber mats and the contact angles of water on the mats submerged in ULSD. PP Flat was a glass slide coated with PP. The remaining materials were fiber mats with the indicated pore and fiber sizes.

\begin{tabular}{|c|c|c|c|c|c|c|c|}
\hline Material & PP Flat & PP105 & PP210 & PP500 & PP1000 & Nylon & PTFE \\
\hline $\begin{array}{c}\text { Edge length of pore (PP) or } \\
\text { area equivalent pore diameter } \\
\text { (nylon, PTFE), } \mu \mathrm{m}\end{array}$ & 0 & 105 & 210 & 500 & 1000 & 785 & 705 \\
\hline Fiber size, $\mu \mathrm{m}$ & NA & 100 & 150 & 300 & 500 & 240 & 260 \\
\hline $\begin{array}{c}\text { Apparent } \\
\text { Water contact angle, deg }\end{array}$ & 145.7 & 148.7 & 144.5 & 143.6 & 136.6 & 139.1 & 142.1 \\
\hline
\end{tabular}

\subsection{Observations from Experiments}

\subsubsection{Side view observations}

Figure 7 shows typical side view images at different moments in time for a water drop moving on a PP500 mat in ULSD. From those images, the advancing and receding angles were estimated. The roughness of the mat structures made the detection of the contact angles difficult, but with careful focusing of the lens the apparent contact angle could be determined. The observed advancing and receding contact angles are summarized in Table 3 . The differences between the advancing and receding angles were within a few degrees; hence the contact line force in Eq.(6) could be neglected. A similar performance was observed for water drops on nylon and PTFE mats. Eq (6) reduced to,

$C_{\text {mat }}=\frac{C_{D}\left(\frac{\pi}{4} d_{L}^{2}\right) \frac{1}{2} \rho_{\text {diesel }}(V-U)^{2}}{\frac{1}{4} \pi d_{c}^{2} \frac{1}{2} \rho_{\text {water }} U^{2}}=\frac{C_{D} d_{L}^{2} \rho_{\text {diesel }}(V-U)^{2}}{d_{c}^{2} \rho_{\text {water }} U^{2}}$ 


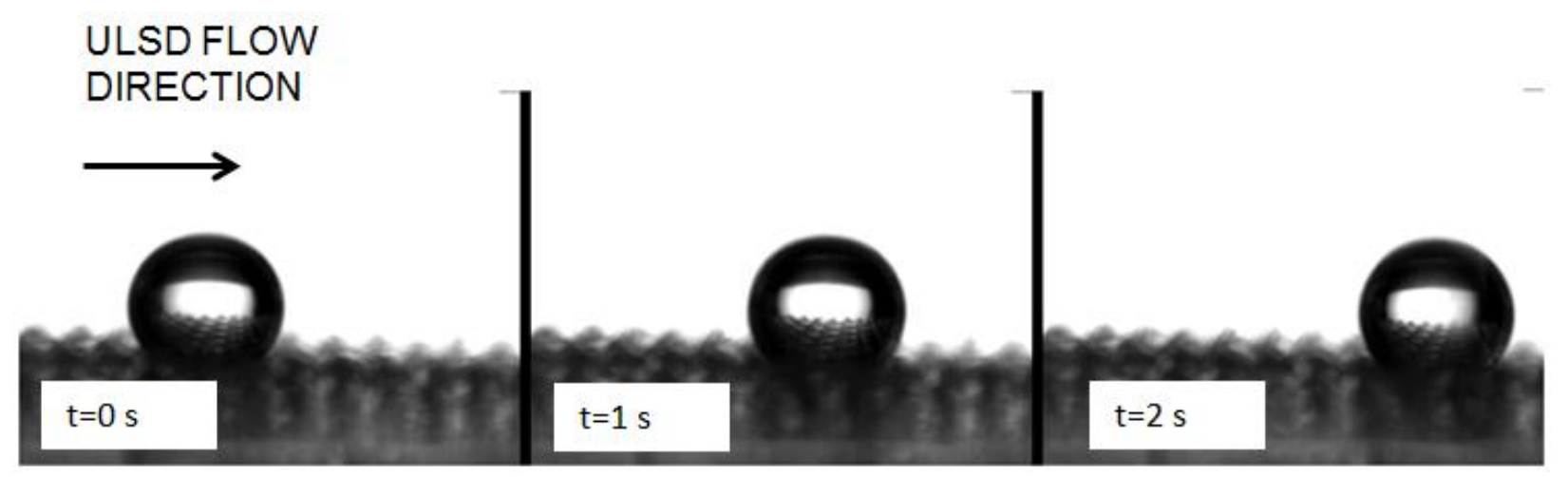

Figure 7. The position of water drops at different times on PP500 mat exposed to flowing ULSD.

Table 3. Summary of advancing and receding angles of water in diesel on PP mat at different times in Figure 7.

\begin{tabular}{|l|c|c|c|}
\hline \multicolumn{1}{|c|}{ Time, $\mathrm{s}$} & 0 & 1 & 2 \\
\hline Advancing, deg & 133.8 & 132.6 & 132.9 \\
\hline Receding, deg & 127.8 & 125.3 & 124.9 \\
\hline
\end{tabular}

\subsubsection{Top view observations}

Figure 8 shows typical images from a top view video recording at different moments in time for a water drop moving on PP210 mat in ULSD. The recording was started at $t=0$ s and the ULSD flow rate was gradually increased to start the movement of the water drop. In this example, the drop started moving at $t=7.25 \mathrm{~s}$, when the ULSD velocity was equal to $\sim 6000 \mu \mathrm{m} / \mathrm{s}$. The ULSD velocity was determined by dividing the volumetric flow rate by the cross sectional area of the channel. From the images, the velocity of drop was calculated to be $\sim 2100 \mu \mathrm{m} / \mathrm{s}$ by dividing the distance moved by the difference in time between the images. The static contact angles (from Table.2) were used to calculate the capillary number in Eq. (11) to correlate the drag coefficient for the movement of the drop on the mat surfaces. 

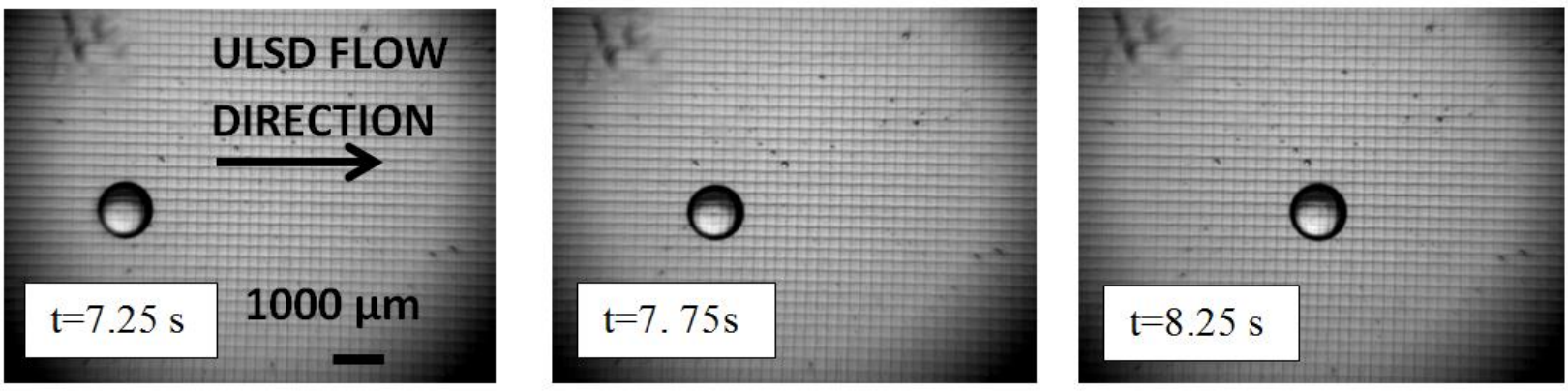

Figure 8. Example images at different moments in times showing the positions of a water drop, on PP210 mat exposed to flowing ULSD at a velocity of $6000 \mu \mathrm{m} / \mathrm{s}$. The drop had a clamshell shape. The calculated velocity of the drop was $2100 \mu \mathrm{m} / \mathrm{s}$

\subsection{Drag coefficient correlation}

The drop movement data were applied in Eq.(17) to calculate the drag coefficients, $C_{m a t}$. The data were fitted to the functional form in Eq.(11). Using regression analysis the best fitted correlation is

$C_{\text {mat }}=0.000877 \operatorname{Re}_{\text {mat }}^{-1.20} C^{-1.18}\left(\frac{d_{A}}{d_{\text {pore }}}\right)^{-1.61}\left(\frac{d_{f}}{d_{\text {pore }}}\right)^{-0.41}(\cos \alpha)^{0.29}$

where the regression analysis calculated the standard errors of the fitted coefficients in Eq.(18) to be $\operatorname{Ln}(A)=-7.03 \pm 2.02, B=-1.20 \pm 0.20, C=-1.18 \pm 0.20, D=-1.61 \pm 0.14, E=-0.41 \pm 0.42$, and $F=-0.29 \pm 0.30$. The $\mathrm{P}$-values for each parameter were $0.0005,3.2 * 10^{-9}, 1.5^{*} 10^{-8}, 7.4^{*} 10^{-27}$, 0.32 , and 0.34 respectively. P-values less than 0.05 are commonly interpreted to mean the parameter is statistically significant. The parameters $B, C, D, E, F$ have very small $\mathrm{P}$-values making them statistically significant. The large P-value and \pm error for $E$ and $F$ shows the parameters $E$ and $F$ were not statistically significant. Also the fitted parameters $B$ and $C$ were nearly the same showing the terms $\operatorname{Re}_{\text {mat }}^{B} C a^{C}$ could be lumped together as $\left(\operatorname{Re}_{\text {mat }} C a\right)^{B}$. The correlation was revised to the form

$$
C_{\text {mat }}=A\left(\operatorname{Re}_{\text {mat }} C a\right)^{B}\left(\frac{d_{A}}{d_{\text {pore }}}\right)^{C}
$$


and the parameters were re-fitted, yielding the best fit correlation to be

$$
C_{\text {mat }}=0.001317\left(\operatorname{Re}_{\text {mat }} C a\right)^{-1.17}\left(\frac{d_{A}}{d_{\text {pore }}}\right)^{-1.68}
$$

where the regression analysis calculated the standard errors of the fitted coefficients in Eq.(20) to be $\operatorname{Ln}(A)=-6.63 \pm 0.30, B=-1.17 \pm 0.03, C=-1.68 \pm 0.12$. The P-values for each parameter were $4.3 * 10^{-65}, 5.3 * 10^{-112}$, and $1.4 * 10^{-35}$ respectively.

Figure 9 shows a plot of the experimental values of $C_{m a t}$ plotted versus the calculated values of $C_{\text {mat }}$ using Eq. (20). The plot follows the $45 \mathrm{deg}$ line as expected with some scatter around the line due to experimental errors. The drag coefficient $C_{\text {mat }}$ spans 5 orders of magnitude from $10^{0}$ to $10^{5}$.

The correlation in Eq.(20) has similarity to the correlation previously reported for barrel-shaped drops on a single fiber where the the exponent values on the $\left(\operatorname{Re}_{m a t} C a\right)$ are both about the magnitude of -1 [15]. However the intercept coefficient 0.001317 is significantly different and for the drop on a single fiber the $\left(\frac{d_{A}}{d_{\text {pore }}}\right)$ is not present.

An interesting physical meaning emerges for the interpretation of the dimensionless drag coefficient, $C_{\text {mat }}$, as recommended by Kasper [22]. If the factional exponent 1.17 is approximated by unity and 1.68 is approximated by 2, then Eq.(20) is approximated by

$$
C_{\text {mat }}=\frac{\text { const }}{\operatorname{Re} C a}\left(\frac{d_{\text {pore }}}{d_{A}}\right)^{2}
$$

Upon rearranging and substituting definitions for $\mathrm{Re}$ and $\mathrm{Ca}$ this becomes

$$
\frac{C_{\text {mat }}}{\text { const }}=\frac{\mu}{\rho U d_{\text {pore }}} \frac{\gamma(1+\cos \theta)}{\mu U}\left(\frac{d_{\text {pore }}}{d_{A}}\right)^{2}=\frac{1}{\rho U^{2}} \frac{\gamma(1+\cos \theta)}{d_{A}} \frac{d_{\text {pore }}}{d_{A}}
$$


Here, the quantities $\rho U^{2}$ and $\frac{\gamma(1+\cos \theta)}{d_{A}}$

represent a dynamic pressure change and capillary pressure. The drag coefficient then becomes

$$
\frac{C_{\text {mat }}}{\text { const }}=\frac{\Delta P_{\text {cap }}}{\Delta P_{d y n}} \frac{d_{\text {pore }}}{d_{A}}
$$

This shows $C_{m a t}$, represents a ratio of capillary to dynamic pressures times the ratio of pore to drop diameters.

Regression fit of the data to Eq.(23) gives a fit of the constant to be const $=0.0075 \pm 0.0003$.

Calculated values for Cmat using Eq.(23) are also plotted on Figure 9. The results show Eq.(23) underestimates the value for Cmat for many of the data points, but gives a reasonably good fit considering it has only one fitted parameter. Data points from Eq.(20) tend to follow the 45 deg line better, but Eq.(20) has three fitted parameters. Defining error as $\mid \ln \left(\mathrm{Cmat}_{\text {calc }} / \mathrm{Cmat}\right.$ expt $) \mid$ the sum of errors of the data using Eq.(20) is 283 and the sum of errors using Eq.(23) is 361 indicating Eq.(20) more closely follows the 45 deg line. In some engineering applications Eq.(23) may give satisfactory estimate of Cmat. 


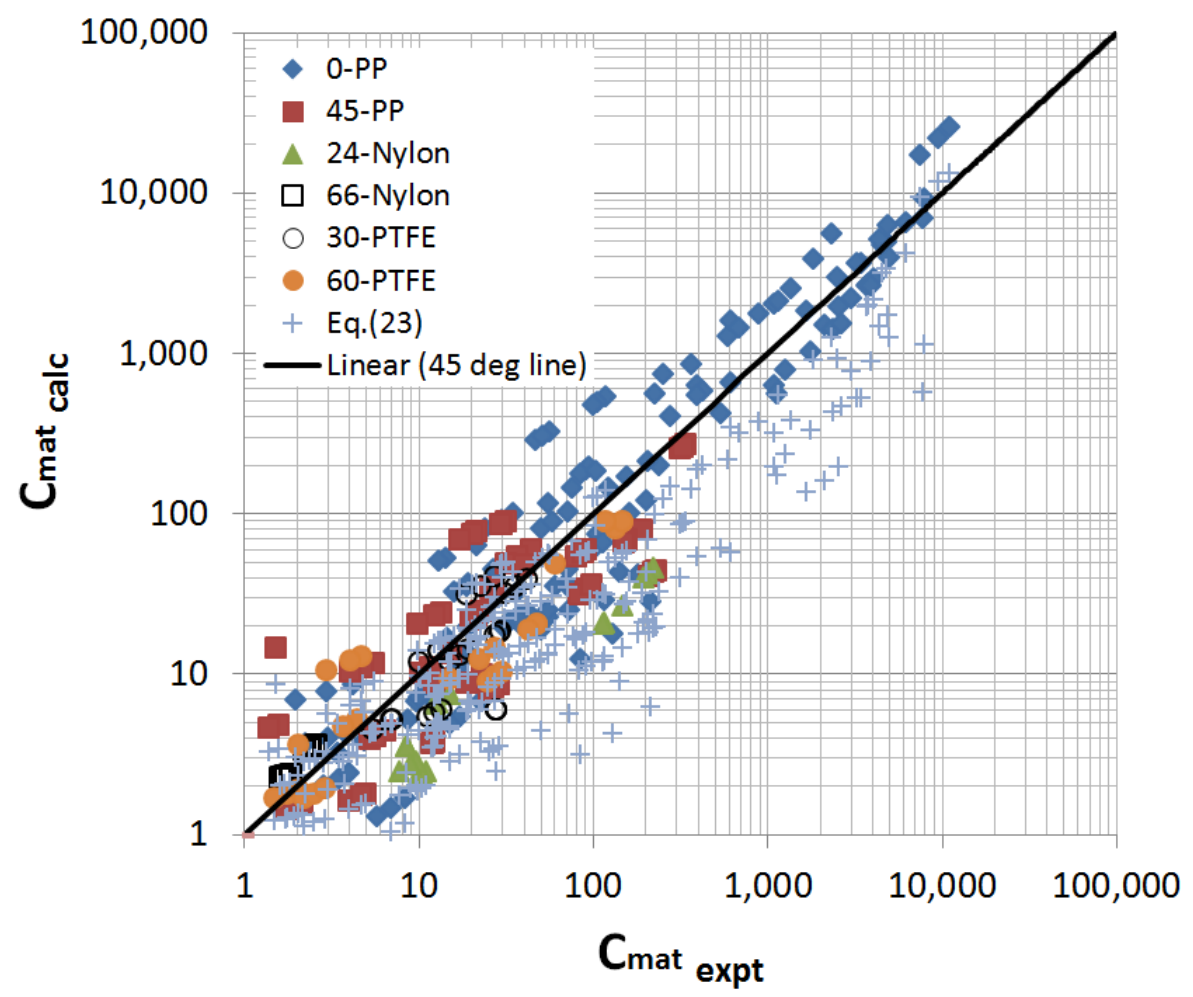

Figure 9 Plot of Cmatcalc calculated from Eq.(20) using the experimental values of ( $\left.\operatorname{Re}_{\text {mat }} C a\right)$ and $\left(d_{A} / d_{\text {pore }}\right)$, versus the experimental value of Cmatexpt. The 45 degree line represents the ideal fit. The legend notation indicates the angles $(0,45,24,66,30,60 \mathrm{deg})$ and the fiber materials (PP, nylon, PTFE).

\subsection{Minimum Reynolds number for drop movement}

The ULSD flow rate was gradually increased to determine the lowest Reynolds number that initiated the drop motion.. Larger drops were easier to move than the smaller drops when exposed to the similar ULSD flow conditions.

The unknown parameters $H, I, J, K, L, M, N, O$ in Eq.(16) were determined by regression of the data from 132 moving drops,

$\operatorname{Re}_{U L S D \min }=0.000162 L a^{0.94}\left(\frac{d_{L}}{d_{\text {pore }}}\right)^{-0.63}$ 
The calculated the standard errors of the fitted coefficients in Eq.(24) to be $\ln (H)=-8.73 \pm 0.67$, $I=0.94 \pm 0.06, K=-0.63 \pm 0.04$. The $P$-values were $2.4 \times 10^{-25}, 2.3 \times 10^{-34}$ and $3.8 \times 10^{-32}$ respectively, indicating the $L a$ and $d_{L} / d_{\text {pore }}$ are parameters were statistically significant.

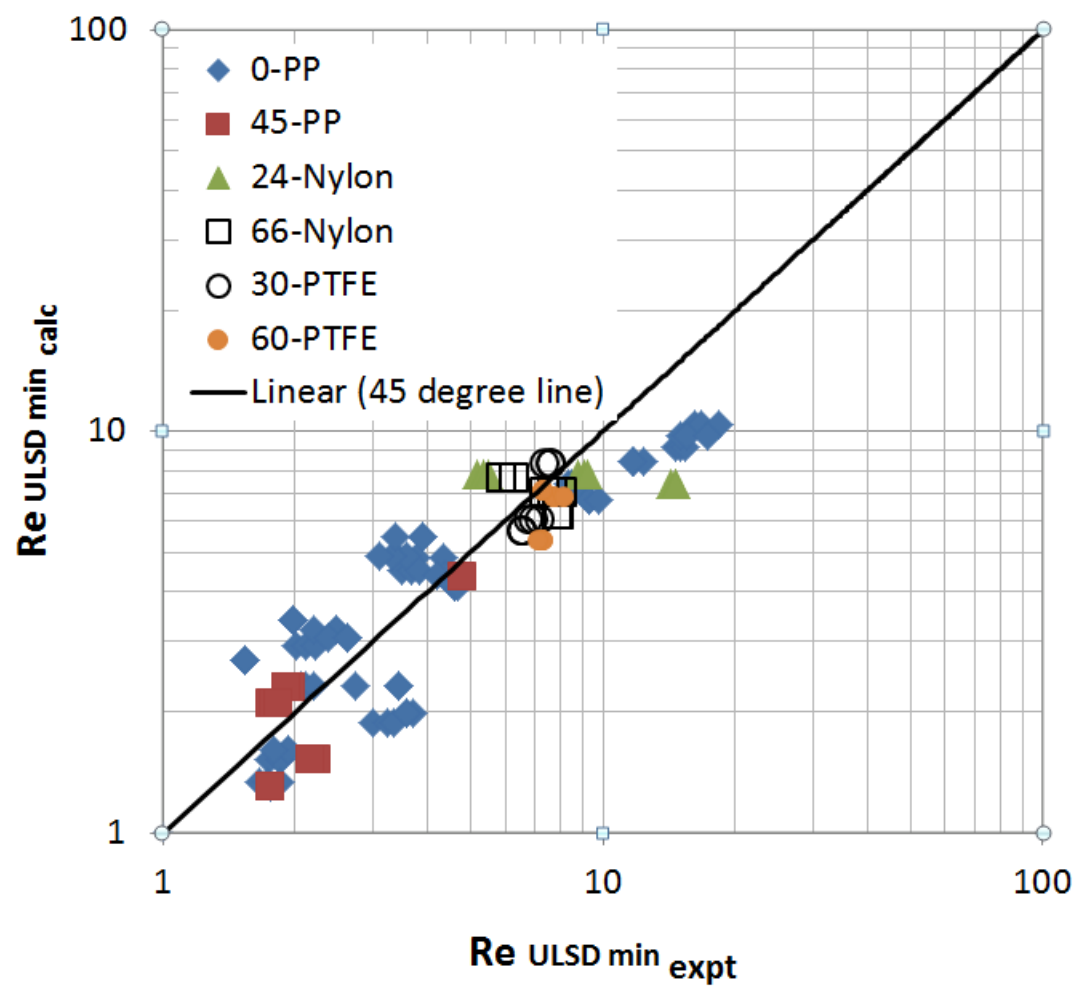

Figure 10 Plot of ReULSD min calc calculated from Eq.(21) using the experimental values of ( $L a)$ and $\left(d_{L} / d_{\text {pore }}\right)$ versus the experimental value of ReULSD min expt . The 45 degree line represents the ideal fit. The legend notation indicates the angles $(0,45,24,66,30,60 \mathrm{deg})$ and the fiber materials (PP, nylon, PTFE).

Figure 10 shows a plot of the experimental values of $R e_{U L S D}$ min plotted versus the calculated values of ReULSD min using Eq. (21). The plot follows the $45 \mathrm{deg}$ line as expected with some scatter around the line due to experimental errors. The plot in Figure 10 has fewer data points than the plot in Figure 9 because only the data of drops that just started moving are plotted in Figure 10, whereas Figure 9 includes data for drops at ULSD flow rates greater than the minimum flow required to initiate movement.

\section{Conclusions}


The movements of clamshell shaped water drops on surfaces of PP, Nylon, and PTFE woven mats were experimentally observed. The drop movements were driven by drag of flowing ULSD fuel across the mat surface. Through dimensional analysis the experimental data were fitted to two correlations. The first correlation estimates the drag coefficient between a drop and fiber mat as the moves on the woven mat surfaces. Two forms of the correlation are provided, one with three fitted parameters and the other with one fitted parameter. The drag coefficient could be used to estimate the average drop velocity. The second correlation provides an estimate of the minimum Reynolds number value needed to initiate drop movement on the mat surfaces.

Future work may extend these correlations to other materials and greater ranges in drop sizes. The results of this work will complement computational studies to calculate and predict motions of drops on fiber mats.

\section{ACKNOWLEDGEMENTS}

This work was supported by the National Science Foundation (CBET 1402336) and by the Coalescence Filtration Nanofibers Consortium at The University of Akron (Ahlstrom, Bekaert, Cummins Filtration, Donaldson, Hollingsworth \& Vose, Parker Hannifin, and SNS Nanofiber Technology LLC).

\section{REFERENCE}

[1] X. Zhang, F. Shi, J. Niu, Y. Jiang, Z. Wang, Superhydrophobic surface: from structural control to functional application, J. Mater. Chem. 18 (2008) 621-633.

[2] S.U. Patel, S.U. Patel, G.G. Chase, Electrospun Superhydrophobic Poly(vinylidene fluorideco-hexafluoropylene) Fibrous Membranes for the Separation of Dispersed Water from Ultra low Sulfur Diesel, Energy Fuels. 27 (2013) 2458-2464.

[3] R.C. Brown, Air Filtration, Pergamon Press, Oxford, 1993.

[4] A. Charvet, Y. Gonthier, A. Bernis, E. Gonze, Filtration of liquids aerosols with a horizontal fibrous filter, Chem. Res. Des. 86 (2008) 569-576. 
[5] G. Viswanadam, G.G. Chase, Water-diesel secondary dispersion separation using superhydrophobic tubes of nanofibers, Sep. Purif. Technol. 104 (2013) 81-88.

[6] S. Madani, A. Amirfazli, Oil drop shedding from solid substrates by a shearing liquid, Colloids and Surfaces A: Physicochem. Eng. Aspects. 441 (2014) 796-806.

[7] P. Roach, N.J. Shirtcliffe, M.L. Newton, Progess in superhydrophobic surface development, Soft Matter. 4(2008) 3404-3407.

[8] Z. Guo, W. Liu, B. Su, Superhydrophobic surface: From natural to biometic to functional, J.Colloid Interface Sci. 353(2011)335-355.

[9] S. Daniel, M. K. Chaudhury, Rectified motion of liquid drops on gradient surfaces induced by vibration, Langmuir, 18 (2002) 3404-3407.

[10] R.S. Subramanian, N. Moumen, J.B. McLaughlin, Motion of a drop on a solid surface due to a wettability gradient, Langmuir. 21 (2005) 11844-11849.

[11] E. Gauthier, T. Hellstern, I.G. Kevrekidis, J. Benziger, Drop detachment and motion on fuel cell electrode materials, Appl. Mater. Interfaces. 4 (2012) 761-771.

[12] X. Sheng, J. Zhang, Directional motion of water drop on ratchet-like superhydrophobic surfaces, Appl. Surf. Sci. 257 (2011) 6811-6816.

[13] B. Bhushan, Y.C. Jung, Wetting study of patterned surfaces for superhydrophobicity, Ultramicroscopy. 107 (2007) 1033-1041.

[14] C. Shin, G.G. Chase, Water-in-oil coalescence in micro-nanofiber composite filters, AICHE J. 50 (2004) 343-350.

[15] J. Fang, M. Davoudi, G.G. Chase, Drop movement along a fiber axis due to pressure driven air flow in a thin slit, Separation and Purification Technology, 140 (2015), 77-83.

[16] S. Dawar, G.G. Chase, Correlations for transverse motion of liquid drops on fibers, Sep. Purif. Technol. 72 (2010) 282-287.

[17] R.S. Subramanian, N. Moumen, J.B. McLaughlin, Motion of a Drop on a Solid Surface Due to a Wettability Gradient, Langmuir. 21 (2005) 11844-11849.

[18] V. Pratap, N. Moumen, R.Shankar. Subramanian, Thermocapillary motion of a liquid drop on a Horizontal solid surface, Langmuir, 24, 5185-5193, 2008.

[19] J.N. Tilton, Fluid and Particle Dynamics, Section 6 in Perry's Chemical

Engineers' Handbook, 8th edition, D.W. Green, R.H. Perry (Eds.), McGraw- 
Hill, New York, 2008.

[20] M. Zlokaranik, Dimensional Analysis and Scale-up in Chemical Engineering, SpringerVerlag, Berlin, 1991.

[21] T. Szirtes, Applied Dimensional Analysis and Modeling, McGraw-Hill, New York, 1998.

[22] G Kasper, Karlsruher Institut für Technologie, Personal correspondence, June 2016.

\section{Nomenclature}

$A$ area of contact between the drop and the mat $\left(\mu \mathrm{m}^{2}\right)$

$A_{d r o p} \quad$ projected area of the drop in the direction of diesel flow $\left(\mu \mathrm{m}^{2}\right)$

$C_{D}$ drag coefficient due to the diesel drag around the drop

$C_{m a t}$ drag coefficient due to the drop on the mat

$\mathrm{Ca}$ Capillary number

$d_{A}$ length of drop measured horizontal to the mat $(\mu \mathrm{m})$

$d_{c}$ diameter of contact area $(\mu \mathrm{m})$

$d_{f} \quad$ diameter of fiber $(\mu \mathrm{m})$

$d_{\text {pore }}$ diameter of pore on the mat $(\mu \mathrm{m})$

$d_{L} \quad$ length of drop measured perpendicular to the mat $(\mu \mathrm{m})$

$F_{\text {cap }}$ capillary force $(\mathrm{N})$

$F_{c l} \quad$ contact line force $(\mathrm{N})$

$F_{D} \quad$ drag force acting on the drop due to diesel flow (N)

$F_{f} \quad$ drag force of moving drop on fiber $(\mathrm{N})$

La Laplace number

$\operatorname{Re}_{U L S D}$ Reynolds number of the diesel

$R e_{\text {mat }}$ Reynolds number of the drop on the mat

$t \quad$ time scale (s) 
$U \quad$ drop velocity $(\mathrm{m} / \mathrm{s})$

$V \quad$ gas velocity $(\mathrm{m} / \mathrm{s})$

\section{Greek symbols}

$\alpha \quad$ angle of one fiber relative to the flow direction

$\gamma_{\text {water }}$ surface tension of the water drop $(\mathrm{N} / \mathrm{m})$

$\mu_{\text {diesel }} \quad$ viscosity of diesel $(\mathrm{Kg} /(\mathrm{m} \mathrm{s}))$

$\mu_{\text {water }} \quad$ viscosity of the water drop $(\mathrm{Kg} /(\mathrm{m} \mathrm{s}))$

$\theta_{a} \quad$ advancing contact angle between the drop and the mat, deg

$\theta \quad$ static contact angle between the drop and the mat, deg

$\theta_{r} \quad$ receding contact angle between the drop and the mat

$\rho_{\text {diesel }}$ density of diesel $\left(\mathrm{Kg} / \mathrm{m}^{3}\right)$

$\rho_{\text {water }}$ density of water $\left(\mathrm{Kg} / \mathrm{m}^{3}\right)$ 




Movement of water drops due to flow of Ultra Low Sulfur Diesel (Top and Side Views) on woven fiber mats of PP, Nylon, PTFE.

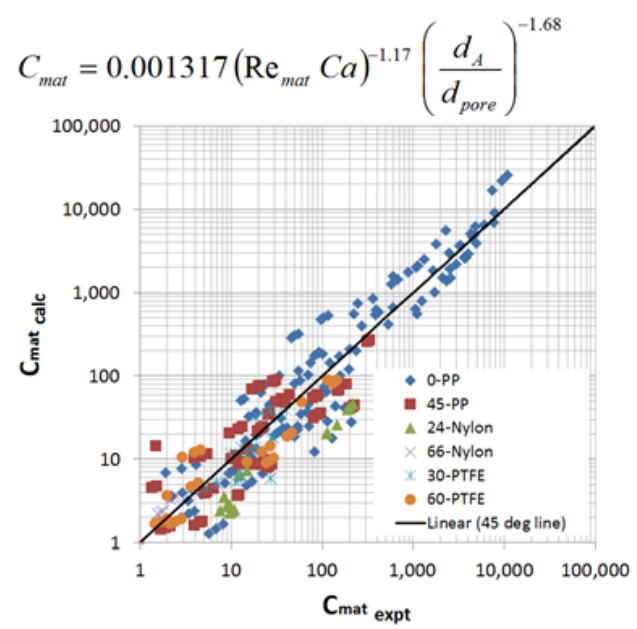

Drag coefficient correlation for predicting rate of drop. 